\title{
Kinetics of Hydrogen Isotope Absorption for Well-Annealed Palladium-Platinum Alloys
}

\author{
Y. Jin, L. Wang, M. Hara* and K. Watanabe \\ Hydrogen Isotope Research Center, University of Toyama, Toyama 930-8555, Japan
}

\begin{abstract}
Absorption kinetics of protium and deuterium for $\mathrm{Pd}, \mathrm{Pd}-4$ at $\% \mathrm{Pt}$ and $\mathrm{Pd}-8$ at $\% \mathrm{Pt}$ were studied in a dilute hydrogen concentration region in a temperature region from 274 to $357 \mathrm{~K}$ by means of constant volume method, where samples were annealed well at $973 \mathrm{~K}$ under vacuum before measurements to remove defects induced by sample preparation. It was found that protium had larger rate constants for adsorption, lower rate constants for desorption and larger diffusion constants than those of deuterium within the examined temperature region. The activation energy of hydrogen absorption for both hydrogen isotopes was almost null for all of the samples used. The activation energy of desorption for deuterium was smaller than that for protium. It was attributed to the difference in the enthalpy of hydrogen absorption into metal in dilute hydrogen concentration region between protium and deuterium. These features and trends observed in the alloying effect were supported by the $a b$ initio calculations for small clusters. [doi:10.2320/matertrans.48.560]
\end{abstract}

(Received September 13, 2006; Accepted January 15, 2007; Published February 25, 2007)

Keywords: palladium alloy, kinetics, isotope effect, hydrogen absorption, hydrogen desorption, diffusion

\section{Introduction}

Attention has been paid to hydrogen absorption by palladium and its alloys with transition elements because they show a considerable large thermodynamic isotope effect on hydrogen absorption. ${ }^{1-3)}$ The property of Pd-Pt alloys is especially useful for the separation of hydrogen isotopes and has been successfully applied for an advanced gas chromatography for hydrogen isotope separation around room temperature without using any replacement gas. ${ }^{4-6)}$ It is reported in our previous papers ${ }^{7,8}$ that the heat of hydrogen absorption as well as hydride formation decreases with increasing platinum-content in the alloy. On the other hand, the thermodynamic isotope effect on hydrogen absorption measured by the ratio of equilibrium pressures between protium and deuterium are almost invariable despite the change in the alloy composition. ${ }^{7,8)}$ It suggests that one can select a proper alloy for operation under a given condition of the chromatograph without changing the magnitude of the isotope effect. 7,8 )

From the other viewpoint, however, since the isotope separation by this system is principally due to the thermodynamic isotope effect, an important factor for separation is to establish absorption equilibrium in a separation column during flowing out of the charged mixture gas of hydrogen isotopes. Therefore, it is required that the rates of absorption and desorption are sufficiently fast and the kinetic isotope effect does not affect the separation efficiency. The kinetic data for hydrogen absorption and desorption are also indispensable to predict the performance of the gas chromatography operated under a given condition. From this viewpoint, the present authors have studied the kinetics of hydrogen absorption by Pd-Pt alloys, ${ }^{9-12)}$ which are considered to be one of the most profitable materials for preparing the separation column.

According to the preliminary studies using a microbalance system, ${ }^{9-12)}$ the absorption rate constant for protium was

*Corresponding author, E-mail: masahara@ctg.u-toyama.ac.jp greater than that for deuterium, and the desorption rate constant as well as the diffusion constant for protium were smaller than those for deuterium. The differences were rather small in comparison with the thermodynamic isotope effect. These rate processes were unexpectedly slow and required rather large activation energies. It was considered due to surface poisoning by residual gases presented in the experimental apparatus. Accordingly, a new apparatus with much lower residual gas pressure was employed to reexamine the rates of absorption of protium and deuterium by Pd-Pt alloys. It was found that the absorption rates of protium and deuterium were one to two orders of magnitude faster than those observed previously. ${ }^{12)}$ This was ascribed to the preparation of cleaner surface state of samples under much better vacuum conditions. The diffusion constants of protium and deuterium obtained by analyzing the absorption curves were significantly difference between previous work ${ }^{12)}$ and the data in leterature ${ }^{13)}$ work. One plausible reason of this observation is considered to lie in the fact that the samples used in the previous work ${ }^{12)}$ were not annealed well at the given activation temperature of $573 \mathrm{~K}$. To examine effect of annealing of Pd-Pt alloys, alloy powders prepared from the same ingots used in the previous work were subjected again to measurements of absorption curves by use of the same apparatus, where the powders were activated at a much higher temperature of $973 \mathrm{~K}$ in the present study.

\section{Experimentals}

Pure $\mathrm{Pd}, \mathrm{Pd}-4$ at $\%$ and $\mathrm{Pd}-8$ at $\% \mathrm{Pt}$ powders smaller than 200 mesh $(<74 \mu \mathrm{m})$ were prepared from the corresponding plates purchased from Tanaka Kikinzoku Kogyo K. K. with the weight of $0.3741 \mathrm{~g}, 0.5089 \mathrm{~g}$ and $0.5196 \mathrm{~g}$, respectively. The purities of those materials were guaranteed to be above 99.9\%. $\mathrm{H}_{2}$ gas was from Nippon Sanso Corporation with purities above $99.9999 \% . \mathrm{D}_{2}$ used in the measurements was refined by $\mathrm{ZrNi}$ alloy from pure $\mathrm{D}_{2}$ gas (It was guaranteed $99.6 \%$ by Nippon Sanso Corporation).

The experimental apparatus used in the present work was 




Fig. 1 X-ray diffraction patterns of Pd-4 at\%Pt powder with and without annealing at $973 \mathrm{~K}$, representing annealing effect.

the same as that described in a previous paper. ${ }^{12)}$ After being loaded in the sample chamber and prior to experiments, all the samples were annealed twice at $973 \mathrm{~K}$ under evacuation with turbo molecular pump with the final pressure around $2.0 \times 10^{-5} \mathrm{~Pa}$. According to X-ray diffraction measurements, diffraction peaks of $\mathrm{Pd}$ and the alloy powders became noticeably sharper owing to the vacuum heating at $973 \mathrm{~K}$ in comparison with those before annealing as shown in Fig. 1. It indicates that the powders prepared by filing the plates were rather rich in defects, but the defects disappeared and the powders were crystallized well by the vacuum heating.

These sample powders were degassed before absorption measurements at $573 \mathrm{~K}$ until the vacuum below $1.3 \times 10^{-5}$ $\mathrm{Pa}$ was attained. Then, the valve above the sample tube was closed and the sample was stabilized at a given experimental temperature by water (water/ice) bath for about 1 hour. Subsequently, a prescribed amount of protium or deuterium was introduced into the standard volume of $V_{1}$. The measurement of gas pressure change started when the valve between the standard volume and the sample tube was opened. The pressure change in the chamber and the average room temperature during the experiment were recorded. The hydrogen absorption was examined in a temperature range of 273 to $358 \mathrm{~K}$. The initial protium/deuterium pressures $P_{\text {ini }}$ were around $67 \mathrm{~Pa}$.

\section{Data Analysis}

The original experimental data are hydrogen pressures declining with time. From the above experimental procedure, one can note that the initial point in pressure change data $(t=0)$ is $P_{\text {ini }} V_{1} / V_{\text {exp }}$, where $V_{\text {exp }}$ is the total volume of the experimental chamber after opening the valve above the sample tube. Since the volumes of the tubes are known, the average $\mathrm{H}$ (hydrogen) concentration in the sample in atomic fraction to metal atom at time $t$ could be estimated from the following equation,

$$
C=\frac{2}{R T_{r} n_{M}}\left(P_{i n i} V_{1}-P_{t} V_{e x p}\right)
$$

where $P_{t}$ is the hydrogen pressure at time $t, T_{r}$ the room temperature, $n_{M}$ the amount of sample in mole. Accordingly, an experimental $C$ - $t$ curve could be obtained from the above conversion.

The data analysis for hydrogen absorption experiments in the previous work, ${ }^{11)}$ where in a vacuum microbalance apparatus with relatively larger chamber was used to keep a constant hydrogen pressure during the measurement, could be adopted in simulating hydrogen absorption in the present system only by replacing the constant pressure with timedependent pressure, $P_{t}$.

The $C$ - $t$ curves obtained were subjected to kinetic analysis. The kinetics of hydrogen absorption are described as

$$
\begin{aligned}
\frac{\partial C}{\partial t} & =D\left(\frac{\partial^{2} C}{\partial r^{2}}+\frac{2}{r} \frac{\partial C}{\partial r}\right) \\
-D\left(\frac{\partial C}{\partial r}\right)_{s} & =k_{a d s} P_{t}\left(N_{S}^{*}-N_{S}\right)^{2}-k_{d e s} N_{s}^{2} \\
& =k_{a b s}^{\prime} P_{t}\left(1-C_{S}\right)^{2}-k_{d e s}^{\prime} C_{S}^{2}
\end{aligned}
$$

where $k_{a d s}^{\prime}$ is the apparent adsorption rate constant, $k_{d e s}^{\prime}$ the desorption rate constant, and $D$ the diffusion constant; $P_{t}, N_{s}^{*}$, $N_{s}$ and $C_{s}$ are the hydrogen pressure at time $t$, the number of active sites for adsorption per unit area, that of adsorbed hydrogen atoms at time $t$ and the corresponding fractional hydrogen concentration, respectively. In this case, the powders were assumed to be spherical; $r$ is the radius of a powder particle. The adsorption rate constant for a given material under a given experimental condition could be evaluated from the initial slope of a $C$ - $t$ curve and the desorption constant was obtained as $k_{d e s}^{\prime}=k_{a d s}^{\prime} / K_{e q}$, where $K_{e q}$ is the equilibrium constant determined experimentally. $K_{e q}$ is expressed with using experimental data as

$$
K_{e q}=\frac{C_{e q}^{2}}{P_{e q}\left(1-C_{e q}\right)^{2}}
$$

where $P_{e q}$ is an equilibrium pressure at a given experimental condition, $C_{e q}$ fraction of hydrogen concentration at equilibrium state. The diffusion constant could be evaluated from trial and error fitting of $C$ - $t$ curves. Details of the analysis were described elsewhere. ${ }^{11,12)}$

\section{Results and Discussion}

\subsection{Absorption curves}

Figure 2 shows, as an example, absorption curves of protium at several different temperatures observed for Pd4 at $\% \mathrm{Pt}$; (a) represents the observed $P$ - $t$ curves and (b) the $C$ - $t$ curves. The dots are experimental data, while lines in (b) are those obtained by simulation following the procedures described above. It is seen that hydrogen absorption under the present conditions was extremely fast and attained to equilibrium within tens of seconds. Hydrogen absorption by $\mathrm{Pd}$ and Pd-8 at\% Pt was also very fast.

In addition, it is seen that the simulation curves agree quite well with those by experimental results, indicating that the set of kinetic parameters as $k_{a d s}^{\prime}, k_{d e s}^{\prime}$ and $D$ can reproduce the observed absorption curves. Similar good agreement was also observed for other samples.

\subsection{Temperature dependence of kinetic parameters}

The Arrhenius plots of kinetic parameters, $k_{a d s}^{\prime}, k_{d e s}^{\prime}$ and $D$, 

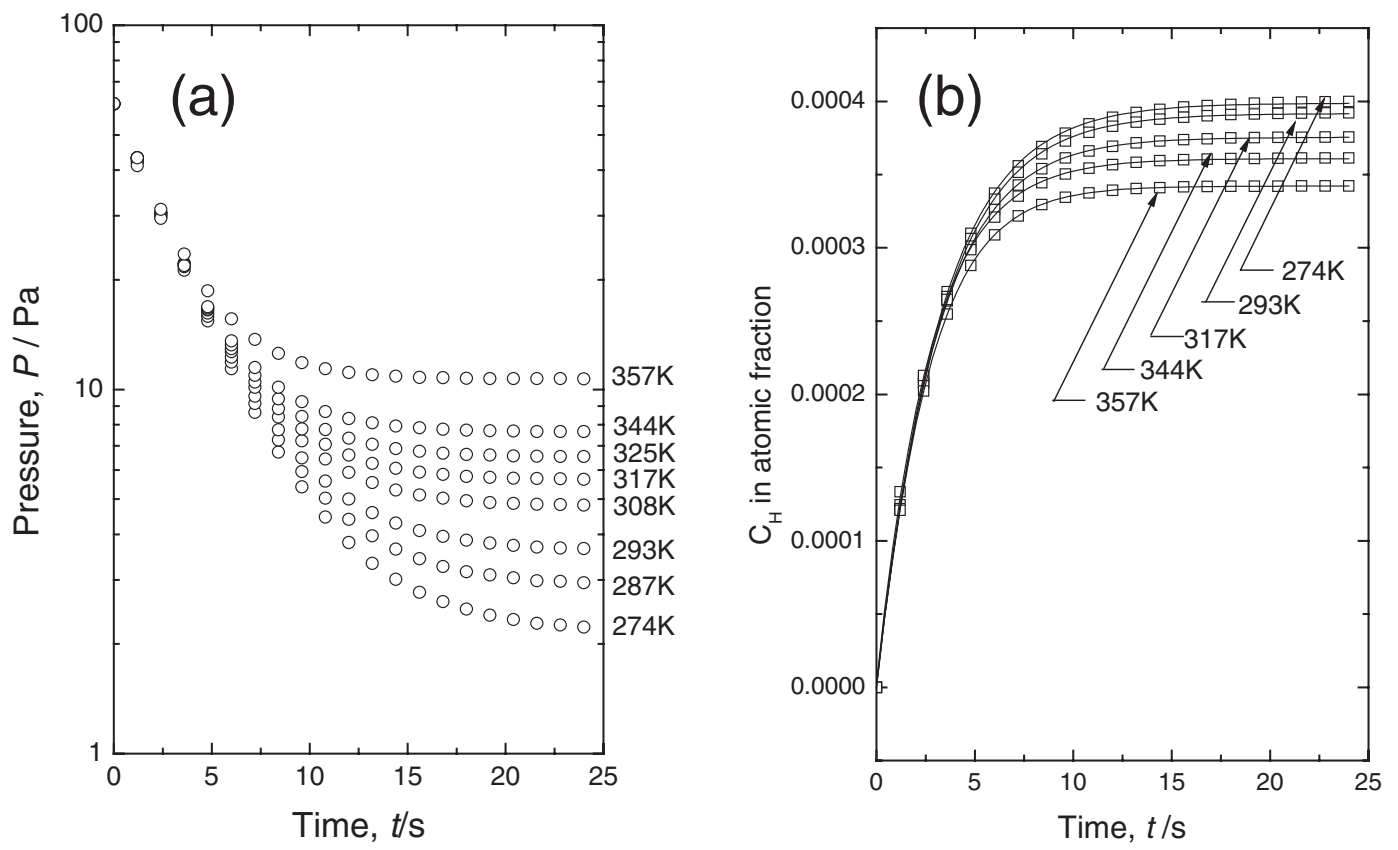

Fig. 2 Hydrogen absorbing behavior of Pd-4 at\% Pt, (a) represents the observed pressure changing and (b) changes in the composition with hydrogen absorption.

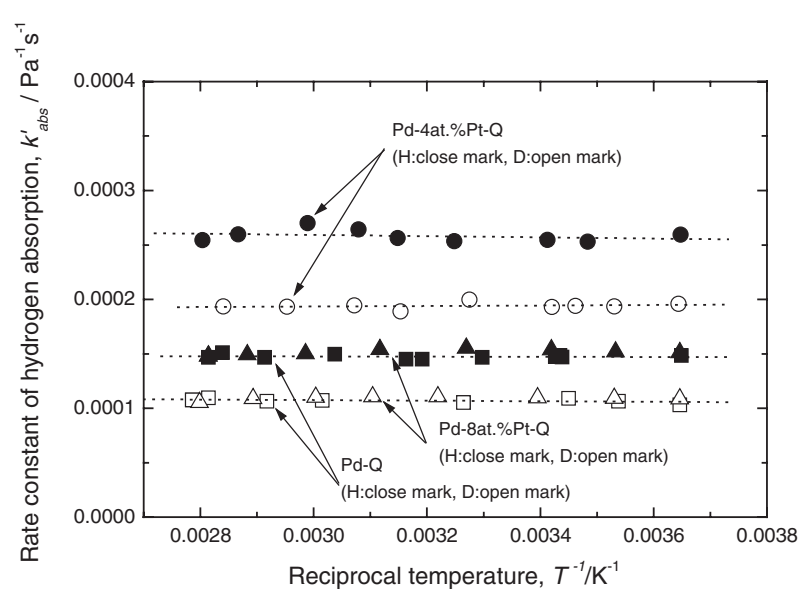

Fig. 3 Temperature dependence of the rate constants of hydrogen absorption.

for both protium and deuterium are shown in Figs. 3, 4 and 5, respectively. It is seen in Fig. 3 that the adsorption rate constants for protium and deuterium for all of the samples were kept almost constant in the temperature range. It indicates that the activation energy for hydrogen adsorption is almost zero. This feature agrees well with the previously reported data obtained for not-well annealed samples. ${ }^{12)}$ The plots of desorption rate constants also showed good linearity for both protium and deuterium for all the samples, but in this case they showed strong temperature dependence. It should be mentioned here that the desorption rates are calculated with using equilibrium constants and absorption rates. The activation energy for desorption obtained from these lines are almost the same as the heat of hydrogen absorption on dilute solution region, $\Delta H$, reported by Yasumatsu. ${ }^{14)}$ This is consistent with the above mentioned results that the

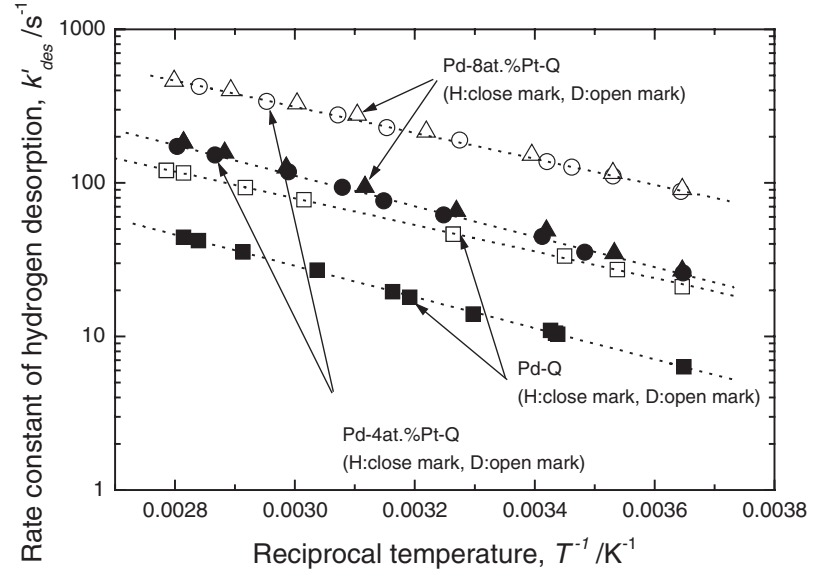

Fig. 4 Temperature dependence of the rate constants of hydrogen desorption.

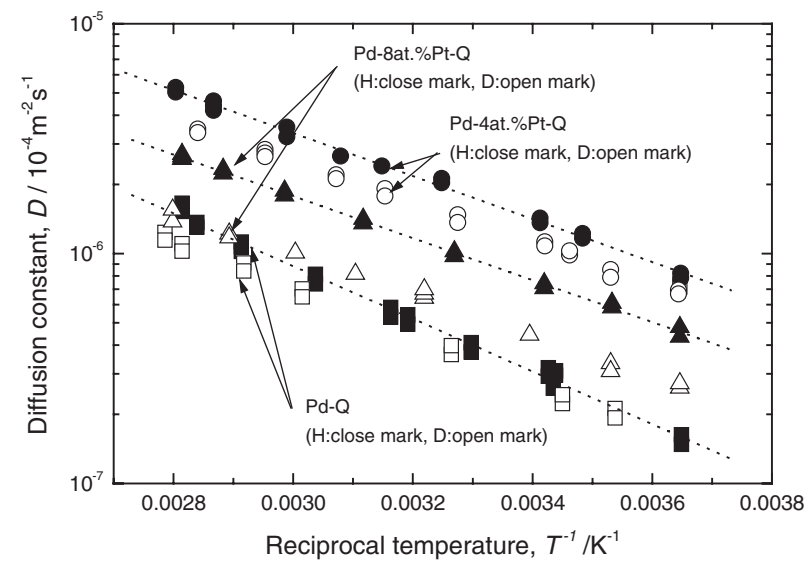

Fig. 5 Temperature dependence of the diffusion constants. 
Table 1 Kinetics parameters of absorption of protium and deuterium for Pd and Pd-Pt alloys.

\begin{tabular}{|c|c|c|c|c|c|c|c|c|c|}
\hline \multirow{2}{*}{\multicolumn{2}{|c|}{$\begin{array}{c}\text { Composition } \\
\text { Source }\end{array}$}} & \multicolumn{3}{|c|}{$\mathrm{Pd}$} & \multicolumn{3}{|c|}{$\mathrm{Pd}-4$ at $\% \mathrm{Pt}$} & \multicolumn{2}{|c|}{$\mathrm{Pd}-8$ at $\% \mathrm{Pt}$} \\
\hline & & This work & Ref. 12 & Ref. 13 & This Work & Ref. 12 & Ref. 11 & This work & Ref. 12 \\
\hline$v_{a b s} /$ & $\mathrm{H}_{2}$ & $1.5 \times 10^{-4}$ & $1.22 \times 10^{-4}$ & - & $2.8 \times 10^{-4}$ & $1.32 \times 10^{-4}$ & - & $1.4 \times 10^{-4}$ & $2.55 \times 10^{-4}$ \\
\hline$\Delta E_{a b s} /$ & $\mathrm{H}_{2}$ & 0.0 & -0.5 & - & 0.2 & 0.6 & 29.1 & -0.3 & 2.4 \\
\hline $\mathrm{kJ} \mathrm{mol}^{-1}\left(\mathrm{H}_{2}, \mathrm{D}_{2}\right)$ & $\mathrm{D}_{2}$ & 0.2 & -0.5 & - & -0.1 & 1.1 & 32.8 & 0.2 & 2.9 \\
\hline$s^{-1}$ & $\mathrm{D}_{2}$ & $3.2 \times 10^{4}$ & $7.41 \times 10^{4}$ & - & $1.1 \times 10^{5}$ & $6.25 \times 10^{5}$ & - & $1.1 \times 10^{5}$ & $8.05 \times 10^{5}$ \\
\hline$\Delta E_{\text {des }} /$ & $\mathrm{H}_{2}$ & 19.4 & 22.1 & - & 19.0 & 28.1 & 48.1 & 19.1 & 26.5 \\
\hline $\mathrm{kJ} \mathrm{mol}^{-1}\left(\mathrm{H}_{2}, \mathrm{D}_{2}\right)$ & $\mathrm{D}_{2}$ & 16.6 & 20.5 & - & 16.2 & 27.4 & 49.0 & 16.1 & 25.2 \\
\hline$-\Delta H_{s o l}^{14)} /$ & $\mathrm{H}_{2}$ & 20.4 & - & - & 19.7 & - & 19.0 & 18.5 & - \\
\hline $\mathrm{kJ} \mathrm{mol}^{-1}\left(\mathrm{H}_{2}, \mathrm{D}_{2}\right)$ & $\mathrm{D}_{2}$ & 16.4 & - & - & 16.7 & - & 16.2 & 15.3 & - \\
\hline$D_{0} /$ & $\mathrm{H}_{2}$ & $2.4 \times 10^{-7}$ & $3.15 \times 10^{-8}$ & $2.90 \times 10^{-7}$ & $2.1 \times 10^{-7}$ & $4.56 \times 10^{-8}$ & $2.0 \times 10^{-8}$ & $9.6 \times 10^{-8}$ & $8.78 \times 10^{-8}$ \\
\hline$\Delta E_{\text {diff }} /$ & $\mathrm{H}_{2}$ & 21.9 & 20.5 & 22.2 & 17.9 & 22.4 & 28.7 & 17.4 & 25.6 \\
\hline$k \mathrm{~mol}^{-1}(H, D)$ & $\mathrm{D}_{2}$ & 19.7 & 20.0 & - & 16.8 & 22.8 & 28.7 & 16.9 & 25.1 \\
\hline
\end{tabular}

activation energy for adsorption is almost zero. The plots of diffusion constants for protium and deuterium also showed good linear relations to the reciprocal of temperature for all the samples. It was observed, as expected, that the value of diffusion constants of protium and deuterium for pure $\mathrm{Pd}$ obtained here agrees fairly well with those reported in literature. ${ }^{13)}$ Set of kinetic parameters, the frequency factor and activation energy, determined from the straight lines (shown for only few for simplicity) in Fig. 3, 4 and 5 are tabulated in Table 1, where some of them are compared with previous observations for convenience.

\subsection{Kinetic isotope effects}

There appears in Fig. 3 a noticeable isotope effect on the adsorption rate constant between protium and deuterium for all of the samples used in the present study. A trend observed is that the rate constant for protium is larger than that for deuterium and the ratio between them is around 1.4. This is due to the difference in the frequency factors, since the activation energies between protium and deuterium are almost same. The size of this isotope effect agrees well with the classical kinetic theory of gases, which predicts the ratio between the number of collisions of protium with a wall per unit time per unit area and that of deuterium to be $\sqrt{m_{D} / m_{H}}$. As for the desorption, it was observed that the desorption rate constant of deuterium was larger than that of protium for all of the samples. There appeared a trend that the frequency factor for desorption of deuterium seems to be almost the same as that of protium. Namely, the isotope effect observed for the desorption rate constant is principally due to the difference in the activation energy. These features are similar to the previous observation for not-well annealed samples. ${ }^{12)}$ On the other hand, the diffusion constant of protium was larger than that of deuterium for all of the samples. This is in contrast with the previous observation, ${ }^{12}$ where the not wellannealed samples showed reverse tendency; namely deuterium gave larger diffusion constant than protium in previous work. ${ }^{12)}$ Nevertheless it is mentioned that the diffusion constants for Pd obtained in the present study are very close to those reported in literature ${ }^{13)}$ and those by the previous

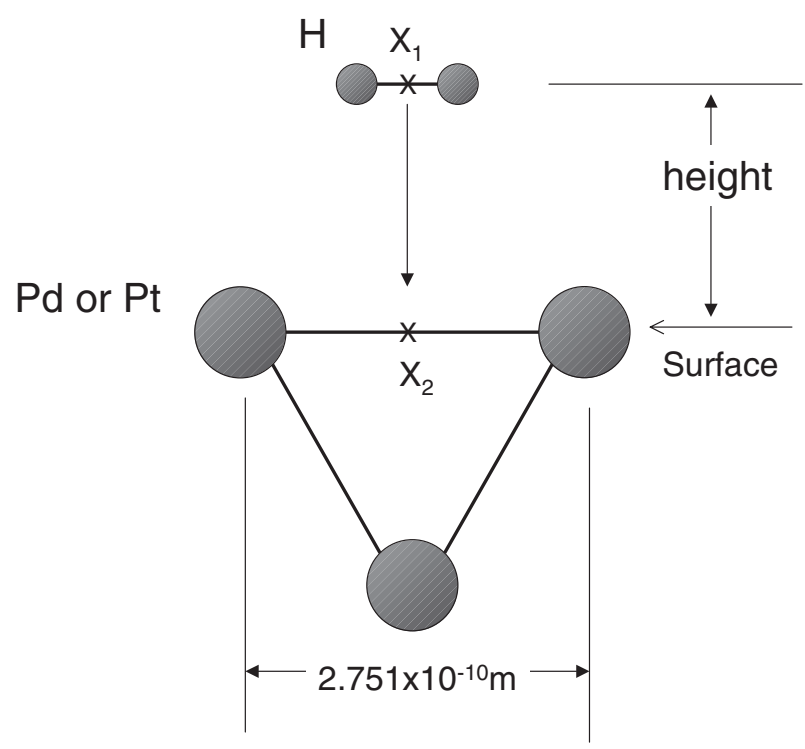

Fig. 6 Cluster model for calculating potential maps.

work was smaller. The larger diffusion constant of protium appeared to be due to the larger frequency factor for protium than that for deuterium; the ratio between them was around 2, inspite that the activation energy for diffusion was evaluated to be larger for protium than deuterium, as seen in Table 1.

\subsection{Alloy effects}

As mentioned above, hydrogen adsorption rate constants are temperature-independent for all the three samples within the tested temperature region; namely the activation energy for adsorption is zero. Therefore, no alloy effect was detected in activation energy for hydrogen adsorption. This feature was examined by ab initio calculation for small clusters using Gaussian 03. ${ }^{15)}$ Figure 6 shows the cluster model used. The cluster is a cut of (111) plane of Pd-fcc structure and hydrogen molecule approaches by keeping the bond axis sidewise to the surface. In this case the calculation was performed by varying distance between two hydrogen atoms, 

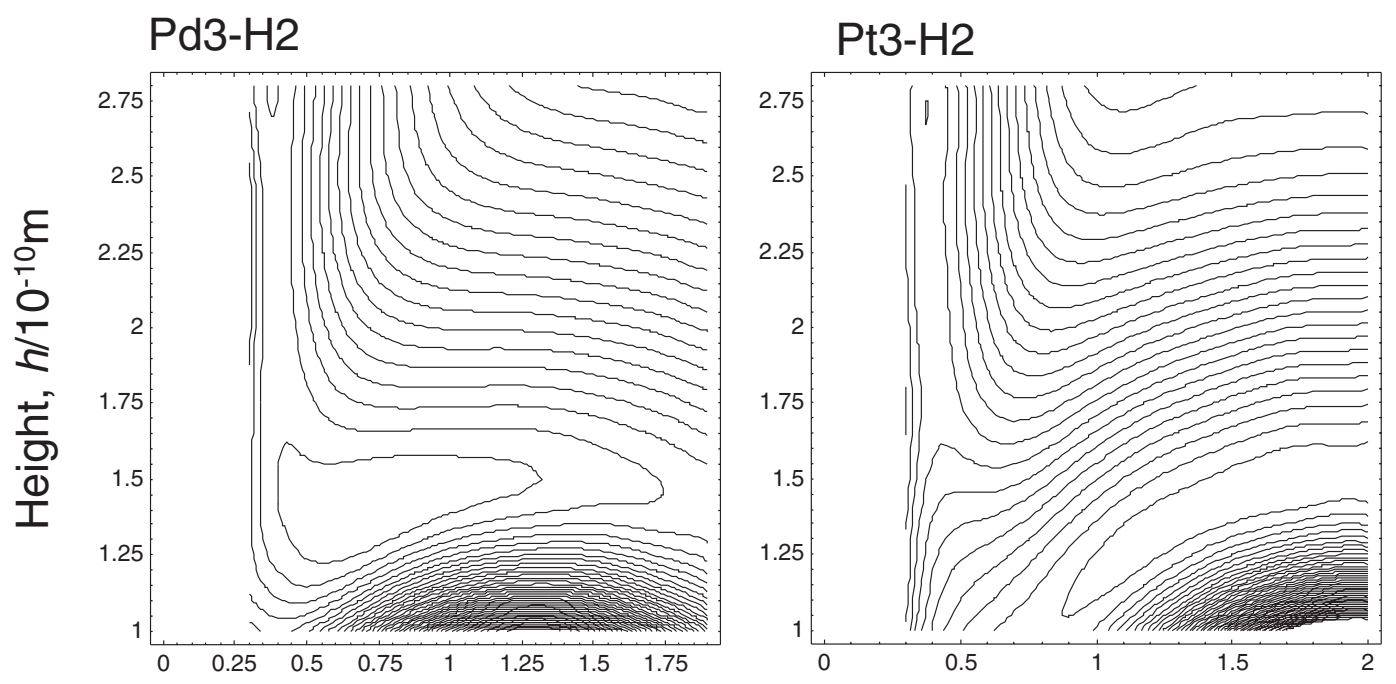

\section{Distance between $\mathrm{H}-\mathrm{H}, \mathrm{d} / 10^{-10} \mathrm{~m}$}

Fig. 7 Potential maps for $\mathrm{Pd} 3+\mathrm{H}_{2}$ or Pt3 $+\mathrm{H}_{2}$.

and that between the centers of $\mathrm{H}-\mathrm{H}$ bond and Me-Me bond (this is assumed to the surface and denoted hereafter height); Me stands for palladium or platinum atom. Details of cluster calculation are described elsewhere. ${ }^{16)}$ Figure 7 shows contour maps of calculated potential surfaces for $\mathrm{Pd} 3-\mathrm{H} 2$ and $\mathrm{Pt} 3-\mathrm{H} 2$ systems. The abscissa of the map is the half distance of $\mathrm{H}-\mathrm{H}$ bond length and ordinate corresponds to the height (distance between $\mathrm{X}_{1}-\mathrm{X}_{2}$ ). The unit of the distance is $10^{-10} \mathrm{~m}$. It is seen in the figure that on approaching of $\mathrm{H}_{2}$ molecule to the palladium surface, there appears no potential barrier for adsorption, indicating that the activation energy for adsorption is zero. This feature is the same for the platinum surface. The results suggest that replacement of palladium atom by platinum gives no appreciable effect on the activation energy of hydrogen adsorption. In addition, there is evidence that palladium atoms should be selectively segregated on the surface for Pd-Pt alloy. ${ }^{17)}$ By assuming PdPt alloy to be an ideal solution, the surface coverage of palladium is estimated to be almost unity for the alloys used in the present study. Accordingly, it is quite plausible that no alloying effect on the activation energy appeared in the present work. In addition, it is expected that the three samples should give the same adsorption rate constant. From this viewpoint, the rate constant for Pd- 4 at $\% \mathrm{Pt}$ appears to be overestimated. This is considered to be due to the net surface area of Pd-4 at\% Pt powder was larger than those of $\mathrm{Pd}$ and Pd-8 at\% Pt powders.

To understand the alloy effect on hydrogen diffusion, Gaussian $03^{15)}$ calculated the potential barriers for hydrogen movement from an octahedral site to a tetrahedral site for a model cluster. Figure 8 shows the model cluster. The cluster was constructed from eight palladium atoms and a hydrogen atom. The cluster is modifications of fcc lattice of Pd-Pt alloy. To inspect hydrogen diffusion in the fcc lattice, the hydrogen atom was initially located at the center of the octahedral site. It was moved from the center of octahedral site to the center of tetrahedral site through the triangle made by number 2, 3 and 4 atoms. The motion of $\mathrm{H}$ atom assumes

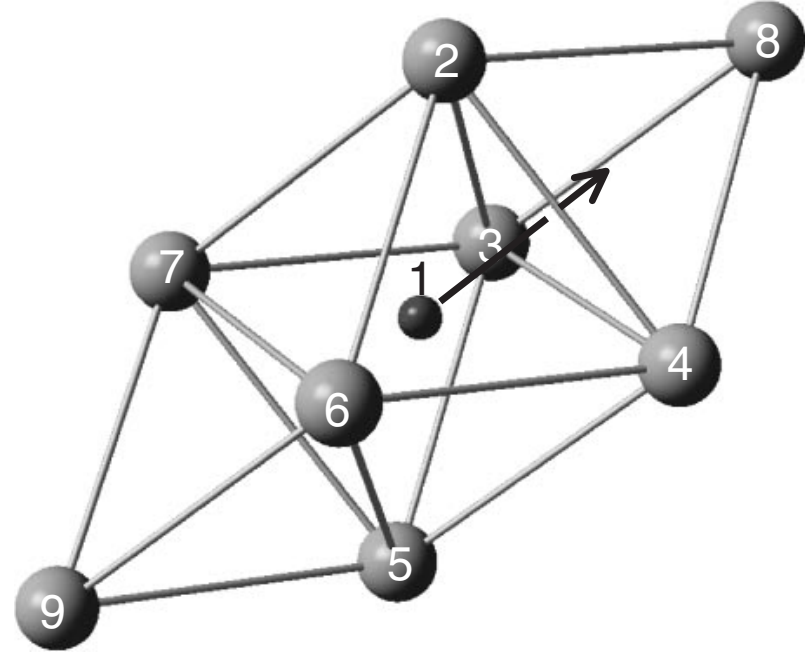

Fig. 8 Cluster model for calculating potential maps of diffusion.

the diffusion of hydrogen atom from an octahedral site to the adjacent octahedral site through the tetrahedral site. A given palladium atom, which constructed the polyhedron, was replaced to a platinum atom to know the change in the potential barrier. Figure 9 shows results of similar calculations for the clusters by Gaussian 03. ${ }^{15}$ ) The abscissa shows the traveling distance of $\mathrm{H}$ atom from the center in $10^{-10} \mathrm{~m}$, and the ordinate the potential energy in $\mathrm{J}_{\text {atom }}{ }^{-1}$. The saddle point arose at the center of the triangle made by $\mathrm{Pd} 2-\mathrm{Pd} 3-$ $\mathrm{Pd} 4$. The activation energy for diffusion corresponds to the energy difference between the octahedral site and the saddle point. It is seen in this figure that replacement of $\mathrm{Pd} 9$ by $\mathrm{Pt}$ causes to move the stable site of $\mathrm{H}$ along $\mathrm{Pd} 9$ direction and to shift up the potential energy, but the effect is much larger at the octahedral site than the saddle point as a result. The activation energy for diffusion is decreased. The platinum replacement of palladium atoms forming the octahedral site forces to lift up the potential energy, indicating this octahedral site is no more valid for hydrogen solution. This 


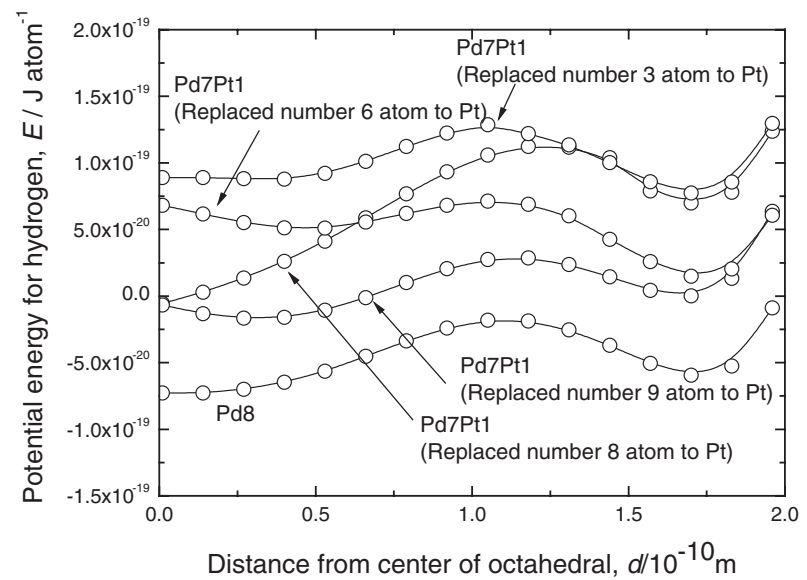

Fig. 9 Potential maps of hydrogen diffusion from center of octahedral site to center of tetrahedral site.

feature coincides well with the observed trend that the activation energy for hydrogen diffusion decreases with increasing platinum content.

\section{Conclusions}

Absorption curves of protium and deuterium by Pd, Pd4 at $\% \mathrm{Pt}$ and $\mathrm{Pd}-8$ at\% Pt were measured in a dilute hydrogen concentration region in a temperature region from 273 to $358 \mathrm{~K}$ by means of constant volume method. Samples were annealed well at $973 \mathrm{~K}$ under vacuum before measurements to remove defects induced by sample preparation. By analyzing the absorption curves, kinetic parameters such as absorption rate, desorption rate and diffusion constants were determined.

Concerning the isotope effect, it was found that the activation energy for absorption of both protium and deuterium was almost null for all of the samples used. On the other hand, the frequency factor for protium was about 1.4 times larger than that of deuterium. Owing to the larger frequency factors, the adsorption rate of protium was greater than that of deuterium in the temperature range studied. As for the desorption, it was found that the rate constant for protium showed smaller than that for deuterium. This was attributed to the fact that the activation energy for protium desorption was greater than that for deuterium, consisting with the difference in the enthalpy change between protium and deuterium solution. Concerning hydrogen diffusion, it was found that the activation energy for protium was larger than that for deuterium, but the frequency factor of deuterium was about half of that of protium. Accordingly, the diffusion constant for protium was greater than that of deuterium.

Concerning the alloying effect, the absence of the alloying effect on the activation energy for absorption was supported by $a b$ initio calculation for small clusters using Gaussian 03 and also by consideration of surface segregation of palladium on Pd-Pt alloy. The results of calculation indicate that a hydrogen molecule can be approaching to a palladium surface without any potential barriers. As for the desorption, the activation energy increased with increasing platinumcontent in the alloy. This feature is consistent with the increase in the enthalpy change for hydrogen absorption with increasing platinum-content in Pd-Pt alloy. It was also observed that the activation energy for hydrogen diffusion decreased with increasing platinum-content in the alloy. This feature was confirmed by small cluster calculation for hydrogen motion by Gaussian 03 .

\section{REFERENCES}

1) R. Lässer and K.-H. Klatt: Phys. Rev. B 28 (1983) 748-758.

2) R. Lässer: Phys. Rev. B 29 (1984) 4765-4768.

3) M. Hara, L. Wan, M. Matsuyama and K. Watanabe: J. Alloy. Compd. 428 (2007) 252-255.

4) K. Watanabe, M. Matsuyama, T. Kobayashi and S. Taguchi: J. Alloy. Compd. 257 (1997) 278-284.

5) K. Watanabe, M. Matsuyama, T. Kobayashi and W. M. Shu: Fusion Engn. Design 39-40 (1998) 1001-1008.

6) S. Ueda, Y. Nanjou, T. Itoh, K. Tatenuma, M. Matsuyama and K. Watanabe: Fusion Sci. and Technol. 41 (2002) 1146-1150.

7) T. Yasumatsu, M. Matsuyama and K. Watanabe: Ann. Rept. Hydrogen Isot. Res. Centr. Toyama Univ. 18 (1998) 81-94.

8) T. Yasumura, J. L. Wan, M. Matsuyama and K. Watanabe: J. Alloy. Compd. 293-295 (1999) 900-907.

9) J. L. Wan, M. Hara, Y. Jin and K. Watanabe: Ann. Rept. Hydrogen Isot. Res. Centr. Toyama Univ. 19 (1999) 9-19.

10) Y. Jin, M. Hara and K. Watanabe: Ann. Rept. Hydrogen Isot. Res. Centr. Toyama Univ. 20 (2000) 21-29.

11) Y. Jin, M. Hara, J. L. Wan, M. Matsuyama and K. Watanabe: J. Alloy. Compd. 340 (2002) 207-213.

12) Y. Jin, M. Hara and K. Watanabe: Ann. Rept. Hydrogen Isot. Res. Centr. Toyama Univ. 22 (2002) 1-10.

13) J. Völkl and G. Alefeld: Hydrogen in Metals I G. Alefeld and J. Völkl Ed. (Springer Verlag, 1978), pp321-348.

14) T. Yasumatsu: Master thesis, Toyama University, 1999.

15) Gaussian 03, Revision B.02, M. J. Frisch, G. W. Trucks, H. B. Schlegel, G. E. Scuseria, M. A. Robb, J. R. Cheeseman, J. A. Montgomery, Jr., T. Vreven, K. N. Kudin, J. C. Burant, J. M. Millam, S. S. Iyengar, J. Tomasi, V. Barone, B. Mennucci, M. Cossi, G. Scalmani, N. Rega, G. A. Petersson, H. Nakatsuji, M. Hada, M. Ehara, K. Toyota, R. Fukuda, J. Hasegawa, M. Ishida, T. Nakajima, Y. Honda, O. Kitao, H. Nakai, M. Klene, X. Li, J. E. Knox, H. P. Hratchian, J. B. Cross, C. Adamo, J. Jaramillo, R. Gomperts, R. E. Stratmann, O. Yazyev, A. J. Austin, R. Cammi, C. Pomelli, J. W. Ochterski, P. Y. Ayala, K. Morokuma, G. A. Voth, P. Salvador, J. J. Dannenberg, V. G. Zakrzewski, S. Dapprich, A. D. Daniels, M. C. Strain, O. Farkas, D. K. Malick, A. D. Rabuck, K. Raghavachari, J. B. Foresman, J. V. Ortiz, Q. Cui, A. G. Baboul, S. Clifford, J. Cioslowski, B. B. Stefanov, G. Liu, A. Liashenko, P. Piskorz, I. Komaromi, R. L. Martin, D. J. Fox, T. Keith, M. A. AlLaham, C. Y. Peng, A. Nanayakkara, M. Challacombe, P. M. W. Gill, B. Johnson, W. Chen, M. W. Wong, C. Gonzalez and J. A. Pople: Gaussian, (Inc., Pittsburgh PA, 2003).

16) L. Wang, R. Hayakawa, Y. Hatano and K. Watanabe: Ann. Rept. Hydrogen Isot. Res. Centr. Toyama Univ. 23 (2003) 20-41.

17) F. L. Williams and D. Nason: Surface Sci. 45 (1974) 377-408. 